\title{
Geometry of effective Hamiltonians
}

\author{
Simen Kvaal* \\ Centre of Mathematics for Applications, \\ University of Oslo, N-0316 Oslo, Norway
}

(Dated: August 13, 2008)

\begin{abstract}
We give a complete geometrical description of the effective Hamiltonians common in nuclear shell model calculations. By recasting the theory in a manifestly geometric form, we reinterpret and clarify several points. Some of these results are hitherto unknown or unpublished. In particular, commuting observables and symmetries are discussed in detail. Simple and explicit proofs are given, and numerical algorithms are proposed, that improve and stabilize common methods used today.
\end{abstract}

PACS numbers: 21.30.Fe,21.60.-n

\footnotetext{
*Electronic address: simen.kvaal@cma.uio.no
} 


\section{INTRODUCTION}

Effective Hamiltonians and interactions are routinely used in shell-model calculations of nuclear spectra [1, 2, 3]. The published mathematical theory of the effective Hamiltonian is complicated and usually focuses on perturbation theoretical aspects, diagram expansions, etc. [4, 5, 6, 7, 8, 9, 10, 11, 12, 13]. In this article, we recast and reinterpret the basic elements of the theory geometrically. We focus on the geometric relationship between the exact eigenvectors $\left|\psi_{k}\right\rangle$ and the effective eigenvectors $\left|\psi_{k}^{\text {eff }}\right\rangle$, both for the usual non-Hermitian Bloch-Brandow effective Hamiltonian [1, 4, 5, 9], and for the Hermitian effective Hamiltonian [6, 11, 14, 15], which we dub the canonical effective Hamiltonian due to its geometric significance. This results in a clear geometric understanding of the de-coupling operator $\omega=Q \omega P$, and a simple proof and characterization of the Hermitian effective Hamiltonian in terms of subspace rotations, in the same way as the non-Hermitian Hamiltonian is characterized by subspace projections.

As a by-product, we obtain a simple and stable numerical algorithm to compute the exact effective Hamiltonian.

The goal of effective interaction theory is to devise a Hamiltonian $H_{\text {eff }}$ in a model space $\mathcal{P}$ of (much) smaller dimension $m$ than the dimension $n$ of Hilbert space $\mathcal{H}$, with $m$ exact eigenvalues of the original Hamiltonian $H=H_{0}+H_{1}$, where $H_{1}$ is usually considered as a perturbation. The model space $\mathcal{P}$ is usually taken as the span of a few eigenvectors $\left\{\left|e_{k}\right\rangle\right\}_{k=1}^{m}$ of $H_{0}$, i.e., the unperturbed Hamiltonian in a perturbational view.

Effective Hamiltonians in $A$-body systems must invariably be approximated (otherwise there would be no need for $H_{\text {eff }}$ ), usually by perturbation theory, but a sub-cluster approximation is also possible [3, 6]. In that case, the exact $a$-body canonical effective Hamiltonian is computed, where $a<A$. From this, one extracts an effective $a$-body interaction and apply it to the $A$-body system. In this case, we present a new algorithm for computing the exact effective interaction that is conceptually and computationally simpler than the usual one which relies on both matrix inversion and square root [3, 11], as the only non-trivial matrix operation is the singular value decomposition (SVD).

The article is organized as follows. In Sec. [I] we introduce some notation and define the singular value decomposition of linear operators and the principal angles and vectors between two linear spaces. In Sec. III we define and analyze the Bloch-Brandow and canonical 
effective Hamiltonians. The main part consists of a geometric analysis of the exact eigenvectors, and forms the basis for the analysis of the effective Hamiltonians. We also discuss the impact of symmetries of the Hamiltonian, i.e., conservation laws. In Sec. IV] we give concrete matrix expressions and algorithms for computing the effective Hamiltonians, and in the canonical case it is, to the author's knowledge, previously unknown. In Sec. $\nabla$ we sum up and briefly discuss the results and possible future projects.

\section{TOOLS AND NOTATION}

\section{A. Linear spaces and operators}

We shall use the Dirac notation for vectors, inner products and operators, in order to make a clear, basis-independent formulation. By $\mathcal{F}, \mathcal{G}$, etc., we denote (finite dimensional) Hilbert spaces, and vectors are denoted by kets, e.g., $|\psi\rangle$, as usual. Our underlying Hilbert space is denoted by $\mathcal{H}$, with $n=\operatorname{dim}(\mathcal{H})$. In general, $n$ is infinite. We shall, however, assume it to be finite. Our results are still valid in the infinite dimensional case if $H$ is assumed to have a discrete spectrum and at least $m$ linearly independent eigenvectors.

We are also given a Hamiltonian $H$, a linear, Hermitian operator (i.e., $H=H^{\dagger}$ ) on $\mathcal{H}$. Its spectral decomposition is defined to be

$$
H=\sum_{k=1}^{n} E_{k}\left|\psi_{k}\right\rangle\left\langle\psi_{k}\right|
$$

Thus, $E_{k}$ and $\left|\psi_{k}\right\rangle$ are the (real) eigenvalues and (orthonormal) eigenvectors, respectively.

We are also given a subspace $\mathcal{P} \subset \mathcal{H}$, called the model space, which in principle is arbitrary. Let $\left\{\left|e_{k}\right\rangle\right\}_{k=1}^{m}$ be an orthonormal basis, for definiteness, viz,

$$
\mathcal{P}:=\operatorname{span}\left\{\left|e_{k}\right\rangle: k=1, \cdots, m\right\}
$$

Let $P$ be its orthogonal projector, i.e.,

$$
P: \mathcal{H} \rightarrow \mathcal{P}, \quad P=\sum_{j=1}^{m}\left|e_{j}\right\rangle\left\langle e_{j}\right|, \quad m=\operatorname{dim}(\mathcal{P}) \leq n,
$$

The basis $\left\{\left|e_{j}\right\rangle\right\}_{j=1}^{m}$ is commonly taken to be eigenvectors for $H_{0}$.

The orthogonal complement of the model space, $\mathcal{Q}=\mathcal{P}^{\perp}$, has the orthogonal projector $Q=1-P$, and is called the excluded space. 
This division of $\mathcal{H}$ into $\mathcal{P}$ and $\mathcal{Q}$ transfers to operators in $\mathcal{H}$. These are in a natural way split into four parts, viz, for an arbitrary operator $A$,

$$
A=(P+Q) A(P+Q)=P A P+P A Q+Q A P+Q A Q
$$

where $P A P$ maps the model space into itself, $Q A P$ maps $\mathcal{P}$ into $\mathcal{Q}$, and so forth. It is convenient to picture this in a block-form of $A$, viz,

$$
A=\left[\begin{array}{ll}
P A P & P A Q \\
Q A P & Q A Q
\end{array}\right] .
$$

\section{B. The singular value decomposition}

A recurrent tool in this work is the singular value decomposition (SVD) of an operator $A: \mathcal{X} \rightarrow \mathcal{Y}$. Here, $p=\operatorname{dim}(\mathcal{X})$ and $q=\operatorname{dim}(\mathcal{Y})$ are arbitrary. Then there exists orthonormal bases $\left\{\left|x_{k}\right\rangle\right\}_{k=1}^{p}$ and $\left\{\left|y_{k}\right\rangle\right\}_{k=1}^{q}$ of $\mathcal{X}$ and $\mathcal{Y}$, respectively, and $r=\min (p, q)$ non-negative real numbers $\sigma_{k}$ with $\sigma_{k} \geq \sigma_{k+1}$ for all $k$, such that

$$
A=\sum_{k=1}^{r} \sigma_{k}\left|y_{k}\right\rangle\left\langle x_{k}\right| .
$$

This is the singular value decomposition (SVD) of $A$, and it always exists. It may happen that some of the basis vectors do not participate in the sum; either if $p \neq q$, or if $\sigma_{k}=0$ for some $k$.

The vectors $\left|x_{k}\right\rangle$ are called right singular vectors, while $\left|y_{k}\right\rangle$ are called left singular vectors. The values $\sigma_{k}$ are called singular values, and $A$ is one-to-one and onto (i.e., nonsingular) if and only if $\sigma_{k}>0$ for all $k$, and $p=q$. The inverse is then

$$
A^{-1}=\sum_{k=1}^{r} \frac{1}{\sigma_{k}}\left|x_{k}\right\rangle\left\langle y_{k}\right|,
$$

as easily verified.

A recursive variational characterization of the singular values and vectors is the following [16]:

$$
\begin{aligned}
& \sigma_{k}=\max _{\substack{|u\rangle \in \mathcal{X},\langle u \mid u\rangle=1 \\
\left\langle u \mid u_{j}\right\rangle=0, j<k}} \max _{\substack{|v\rangle \in \mathcal{Y},\langle v \mid v\rangle=1 \\
\left\langle v \mid v_{j}\right\rangle=0, j<k}} \operatorname{Re}\langle v|A| u\rangle \\
&=:\left\langle v_{k}|A| u_{k}\right\rangle .
\end{aligned}
$$


The latter equality implicitly states that the maximum is actually real. The SVD is very powerful, as it gives an interpretation and representation of any linear operator $A$ as a simple scaling with respect to one orthonormal basis, and then transformation to another. The singular vectors are not unique, but the singular values are.

\section{Principal angles and vectors}

Important tools for comparing linear subspaces $\mathcal{F}$ and $\mathcal{G}$ of $\mathcal{H}$ are the principal angles and principal vectors [17, 18]. The principal angles generalize the notion of angles between vectors to subspaces in a natural way. They are also called canonical angles. Assume that

$$
p=\operatorname{dim}(\mathcal{F}) \geq q=\operatorname{dim}(\mathcal{G}) \geq 1
$$

(If $p<q$, we simply exchange $\mathcal{F}$ and $\mathcal{G}$.) Then, $q$ principal angles $\theta_{k} \in[0, \pi / 2]$, with $\theta_{k} \leq \theta_{k+1}$ for all $k$, and the left and right principal vectors $\left|\xi_{k}\right\rangle \in \mathcal{F}$ and $\left|\eta_{k}\right\rangle \in \mathcal{G}$ are defined recursively through

$$
\begin{aligned}
\cos \theta_{k} & =\max _{\substack{|\xi\rangle \in \mathcal{F},\langle\xi \mid \xi\rangle=1 \\
\left\langle\xi \mid \xi_{j}\right\rangle=0, j<k}} \max _{\substack{|\eta\rangle \in \mathcal{G},\langle\eta \mid \eta\rangle=1 \\
\left\langle\eta \mid \eta_{j}\right\rangle=0, j<k}} \operatorname{Re}\langle\xi \mid \eta\rangle \\
& =:\left\langle\xi_{k} \mid \eta_{k}\right\rangle .
\end{aligned}
$$

Again, the last equality implicitly states that the maximum actually is real. One sees that $\theta_{k}$ is the angle between $\left|\xi_{k}\right\rangle \in \mathcal{F}$ and $\left|\eta_{k}\right\rangle \in \mathcal{G}$.

It is evident from Eqns. (21) and (3) that the principal angles and vectors are closely related to the SVD. Indeed, if we consider the product of the orthogonal projectors $P_{\mathcal{F}}$ and $P_{\mathcal{G}}$ and compute the SVD, we obtain

$$
P_{\mathcal{F}} P_{\mathcal{G}}=\sum_{k=1}^{p}\left|\xi_{k}\right\rangle\left\langle\xi_{k}\left|\sum_{j=1}^{q}\right| \eta_{k}\right\rangle\left\langle\eta_{k}\left|=\sum_{k=1}^{q} \cos \theta_{k}\right| \xi_{k}\right\rangle\left\langle\eta_{k}\right|,
$$

where we extended the orthonormal vectors $\left\{\left|\xi_{k}\right\rangle\right\}_{k=1}^{q}$ with $p-q$ vectors into a basis for $\mathcal{F}$, which is always possible. This equation in particular implies the additional orthogonality relation $\left\langle\xi_{j} \mid \eta_{k}\right\rangle=\delta_{j, k} \cos \theta_{k}$ on the principal vectors.

The principal vectors constitute orthonormal bases that should be rotated into each other if the spaces were to be aligned. Moreover, the rotations are by the smallest angles possible. 


\section{EFFECTIVE HAMILTONIANS}

\section{A. Similarity transforms}

The goal of the effective Hamiltonian is to reproduce exactly $m$ of the eigenvalues, and (necessarily) approximately $m$ of the eigenvectors. We shall assume that the first $m$ eigenpairs $\left(E_{k},\left|\psi_{k}\right\rangle\right), k=1, \ldots, m$, defines these. We define the space $\mathcal{E}$ as

$$
\mathcal{E}:=\operatorname{span}\left\{\left|\psi_{k}\right\rangle: k=1, \cdots, m\right\}
$$

The orthogonal projector $P^{\prime}$ onto $\mathcal{E}$ is

$$
P^{\prime}=\sum_{k=1}^{m}\left|\psi_{k}\right\rangle\left\langle\psi_{k}\right| .
$$

We denote by $\left(E_{k},\left|\psi_{k}^{\text {eff }}\right\rangle\right), k=1, \ldots, m$, the effective Hamiltonian eigenvalues and eigenvectors. Of course, the $\left|\psi_{k}^{\text {eff }}\right\rangle \in \mathcal{P}$ must constitute a basis for $\mathcal{P}$, but not necessary an orthonormal basis. Geometrically, we want $\left|\psi_{k}^{\text {eff }}\right\rangle$ to be as close as possible to $\left|\psi_{k}\right\rangle$, i.e., we want $\mathcal{E}$ to be as close to $\mathcal{P}$ as possible.

Let $\left|\widetilde{\psi_{k}^{\text {eff }}}\right\rangle$ be the bi-orthogonal basis, i.e., $\left\langle\widetilde{\psi_{j}^{\text {eff }}} \mid \psi_{k}^{\text {eff }}\right\rangle=\delta_{j, k}$, so that

$$
P=\sum_{k=1}^{m}\left|e_{k}\right\rangle\left\langle e_{k}\left|=\sum_{k=1}^{m}\right| \psi_{k}^{\mathrm{eff}}\right\rangle\left\langle\widetilde{\psi_{k}^{\mathrm{eff}}}\right| .
$$

The spectral decomposition of $H_{\text {eff }}$ becomes

$$
H_{\mathrm{eff}}=\sum_{k=1}^{m} E_{k}\left|\psi_{k}^{\mathrm{eff}}\right\rangle\left\langle\widetilde{\psi_{k}^{\mathrm{eff}}}\right| .
$$

Since $H_{\mathrm{eff}}$ is to have eigenvalues identical to $m$ of those of $H$, and since $H_{\text {eff }}$ operates only in $\mathcal{P}$, we may relate $H_{\text {eff }}$ to $H$ through a similarity transform, viz,

$$
H_{\mathrm{eff}}=P \tilde{H} P=P\left(e^{-S} H e^{S}\right) P,
$$

where $\exp (S) \exp (-S)=I$. Any invertible operator has a logarithm, so Eqn. (5) is completely general.

Now, $H_{\text {eff }}=P \tilde{H} P$ is an effective Hamiltonian only if the Bloch equation

$$
Q \tilde{H} P=Q e^{-S} H e^{S} P=0
$$




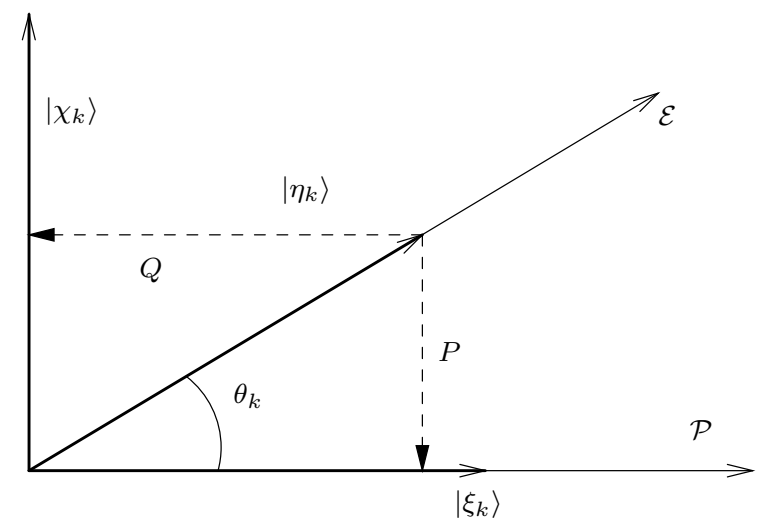

FIG. 1: Plane spanned by $\left|\xi_{k}\right\rangle$ and $\left|\eta_{k}\right\rangle$. Action of projectors $P$ and $Q$ on $\left|\eta_{k}\right\rangle$ indicated

is satisfied [8], since $\mathcal{P}$ is then invariant under the action of $\tilde{H}$. The eigenvectors of $H_{\text {eff }}$ are now given by

$$
\left|\psi_{k}^{\mathrm{eff}}\right\rangle=e^{-S}\left|\psi_{k}\right\rangle \in \mathcal{P}, \quad k=1, \cdots, m .
$$

Thus, an effective Hamiltonian can now be defined for every $S$ such that Eqn. (6) holds. It is readily seen that $\tilde{H}=\tilde{H}^{\dagger}$ if and only if $S$ is skew-Hermitian, i.e., that $S^{\dagger}=-S$. There is still much freedom in the choice of exponent $S$. Indeed, given any invertible operator $A$ in $\mathcal{P}, A^{-1} H_{\text {eff }} A$ is a new effective Hamiltonian with the same effective eigenvalues as $H_{\text {eff }}$, and $\left|\psi^{\mathrm{eff}}\right\rangle=A^{-1} \exp (-S)|\psi\rangle$.

\section{B. Geometry of the model space}

We will benefit from a detailed discussion of the spaces $\mathcal{E}$ and $\mathcal{P}$ before we discuss the Bloch-Brandow and the canonical effective Hamiltonians in detail.

Since $\operatorname{dim}(\mathcal{P})=\operatorname{dim}(\mathcal{E})=m$, the closeness of the effective and exact eigenvectors can be characterized and measured by the orientation of $\mathcal{E}$ relative to $\mathcal{P}$ in $\mathcal{H}$, using $m$ canonical angles $\theta_{k}$ and principal vectors $\left|\eta_{k}\right\rangle \in \mathcal{E}$ and $\left|\xi_{k}\right\rangle \in \mathcal{P}$. Recall, that $\cos \theta_{k}=\left\langle\xi_{k} \mid \eta_{k}\right\rangle$ and that the angles $\theta_{k} \in[0, \pi / 2]$ were the smallest possible such that the principal vectors are the orthonormal bases of $\mathcal{P}$ and $\mathcal{E}$ that are closest to each other.

We now define the unitary operator $Z=\exp (G)$ that rotates $\mathcal{P}$ into $\mathcal{E}$ according to this description, i.e., we should have $Z\left|\xi_{k}\right\rangle=\left|\eta_{k}\right\rangle$. In Fig. 1 the plane spanned by $\left|\eta_{k}\right\rangle$ and $\left|\xi_{k}\right\rangle$ if $\theta_{k}>0$ is depicted. Recall, that $\left\langle\xi_{j} \mid \eta_{k}\right\rangle=\cos \theta_{j} \delta_{j, k}$. Note that $\left|\xi_{k}\right\rangle=\left|\eta_{k}\right\rangle$ if and only if $\theta_{k}=0$, and the plane degenerates into a line. If $\theta_{k}>0$, the vector $\left|\chi_{k}\right\rangle$ is defined so that it 
together with $\left|\xi_{k}\right\rangle$ is an orthonormal basis for the plane, viz,

$$
\left|\eta_{k}\right\rangle=P\left|\eta_{k}\right\rangle+Q\left|\eta_{k}\right\rangle=\cos \left(\theta_{k}\right)\left|\xi_{k}\right\rangle+\sin \left(\theta_{k}\right)\left|\chi_{k}\right\rangle
$$

where

$$
\left|\chi_{k}\right\rangle=\frac{Q\left|\eta_{k}\right\rangle}{\left\langle\eta_{k}|Q| \eta_{k}\right\rangle^{1 / 2}} .
$$

Thus, $\left\{\left|\chi_{k}\right\rangle\right\} \cup\left\{\left|\xi_{k}\right\rangle\right\}$ is an orthonormal basis for $\mathcal{P} \oplus \mathcal{E}$, whose dimension is $2 m-n_{z}$, where $n_{z}$ is the number of $\theta_{k}=0$. The set $\left\{\left|\chi_{k}\right\rangle: \theta_{k}>0, k=1, \cdots, m\right\}$ is an orthonormal basis for $Q \mathcal{E}$ which contains $Q\left|\psi_{k}\right\rangle$ for all $k=1, \cdots, m$.

The operator $Z$ is now defined as a rotation in $\mathcal{P} \oplus \mathcal{E}$, i.e., by elementary trigonometry,

$$
\begin{aligned}
Z\left|\xi_{k}\right\rangle & :=\left|\eta_{k}\right\rangle \\
Z\left|\eta_{k}\right\rangle & :=2 \cos \theta_{k}\left|\eta_{k}\right\rangle-\left|\xi_{k}\right\rangle
\end{aligned}
$$

In terms of the orthonormal basis, we obtain a manifest planar rotation for each $k$, i.e.,

$$
\begin{aligned}
Z\left|\chi_{k}\right\rangle & =\cos \theta_{k}\left|\chi_{k}\right\rangle-\sin \theta_{k}\left|\xi_{k}\right\rangle \\
Z\left|\xi_{k}\right\rangle & =\sin \theta_{k}\left|\chi_{k}\right\rangle+\cos \theta_{k}\left|\xi_{k}\right\rangle .
\end{aligned}
$$

On the rest of the Hilbert space, $\mathcal{H} \ominus(\mathcal{P} \oplus \mathcal{E}), Z$ is the identity. The operator $Z$ implements the so-called direct rotation [19] of $\mathcal{P}$ into $\mathcal{E}$. From Eqn. (11) we obtain

$$
\begin{aligned}
Z:= & I+\sum_{k=1}^{m}\left[\cos \left(\theta_{k}\right)-1\right]\left(\left|\chi_{k}\right\rangle\left\langle\chi_{k}|+| \xi_{k}\right\rangle\left\langle\xi_{k}\right|\right) \\
& +\sum_{k=1}^{m} \sin \left(\theta_{k}\right)\left(\left|\chi_{k}\right\rangle\left\langle\xi_{k}|-| \xi_{k}\right\rangle\left\langle\chi_{k}\right|\right)
\end{aligned}
$$

It is instructive to exhibit the Lie algebra element $G \in s u(n)$ such that $Z=\exp (G) \in$ $S U(n)$. Since we have Eqn. (11), is is easy to do this. Indeed, taking the exponential of

$$
G=\sum_{k=1}^{m} \theta_{k}\left(\left|\chi_{k}\right\rangle\left\langle\xi_{k}|-| \xi_{k}\right\rangle\left\langle\chi_{k}\right|\right),
$$

by summing the series for $\sin (\theta)$ and $\cos (\theta)$, we readily obtain $Z=\exp (G)$, the desired result. Moreover, observe that the $k$ 'th term in Eqn. (13) commutes with the $j$ 'th term, so, $\exp (G)$ is exhibited as a sequence of commuting rotations using the canonical angles $\theta_{k}$. 


\section{The Bloch-Brandow effective Hamiltonian and the decoupling operator}

For reference, we review some properties of the Bloch-Brandow effective Hamiltonian, which we denote by $H_{\text {eff }}^{\mathrm{BB}}[1,4,45,9]$. The effective eigenvectors $\left|\psi_{k}^{\mathrm{eff}}\right\rangle$ are defined by

$$
\left|\psi_{k}^{\mathrm{eff}}\right\rangle:=P\left|\psi_{k}\right\rangle:=\left|P \psi_{k}\right\rangle
$$

Since $\left|P \psi_{k}\right\rangle$ are the orthogonal projections of $\left|\psi_{k}\right\rangle$ onto $\mathcal{P}$, we deduce that the Bloch-Brandow effective eigenvectors are the closest possible to the exact model space eigenvectors. In this sense, the Bloch-Brandow effective Hamiltonian is the optimal choice.

It is obvious that $H_{\mathrm{eff}}^{\mathrm{BB}}$ is non-Hermitian, as rejecting the excluded space eigenvector components renders the effective eigenvectors non-orthonormal, i.e.,

$$
\left\langle\psi_{j}^{\mathrm{eff}} \mid \psi_{k}^{\mathrm{eff}}\right\rangle=\delta_{j, k}-\left\langle\psi_{j}|Q| \psi_{k}\right\rangle \neq \delta_{j, k}
$$

In terms of similarity transforms, we obtain $H_{\mathrm{eff}}^{\mathrm{BB}}$ by setting $S=\omega$, the so-called de-coupling operator or correlation operator $[1,11]$. It is defined by $\omega=Q \omega P$ and the equation

$$
\omega P\left|\psi_{k}\right\rangle:=Q\left|\psi_{k}\right\rangle
$$

Again, for this to be a meaningful definition, $\left\{\left|P \psi_{k}\right\rangle\right\}_{k=1}^{m}$ must be a basis for $\mathcal{P}$.

Since $\omega^{2}=0, \exp ( \pm \omega)=1 \pm \omega$, and Eqn. (7) becomes

$$
e^{-\omega}\left|\psi_{k}\right\rangle=(1-\omega)\left|\psi_{k}\right\rangle=(1-Q)\left|\psi_{k}\right\rangle=\left|P \psi_{k}\right\rangle
$$

For $H_{\text {eff }}^{\mathrm{BB}}$ we thus obtain

$$
H_{\mathrm{eff}}^{\mathrm{BB}}=P e^{-\omega} H e^{\omega} P=P H(P+\omega) .
$$

After this initial review, we now relate $\omega$ to the geometry of $\mathcal{E}$ and $\mathcal{P}$. The SVD of $\omega$ is readily obtainable by expanding the principal vectors $\left\{\left|\eta_{k}\right\rangle\right\}_{j=1}^{m}$ in the $m$ eigenvectors $\left\{\left|\psi_{k}\right\rangle\right\}_{k=1}^{m}$, sets which both constitute a basis for $\mathcal{E}$, and inserting in Eqn. (15). We have

$$
\begin{aligned}
Q\left|\eta_{k}\right\rangle & =\sum_{j=1}^{m} Q\left|\psi_{j}\right\rangle\left\langle\psi_{j} \mid \eta_{k}\right\rangle \\
& =\sum_{j=1}^{m} \omega P\left|\psi_{j}\right\rangle\left\langle\psi_{j} \mid \eta_{k}\right\rangle=\omega P\left|\eta_{k}\right\rangle
\end{aligned}
$$


that is,

$$
\omega\left(\cos \theta_{k}\left|\xi_{k}\right\rangle\right)=\sin \theta_{k}\left|\chi_{j}\right\rangle
$$

The result is

$$
\omega=\sum_{k=1}^{m} \tan \theta_{k}\left|\chi_{k}\right\rangle\left\langle\xi_{k}\right|,
$$

which is the SVD of $\omega$. The operator $\omega$ is thus exhibited as an operator intimately related to the principal angles and vectors of $\mathcal{P}$ and $\mathcal{E}$ : It transforms the principal vectors of $\mathcal{P}$ into an orthonormal basis for $Q \mathcal{E}$, with coefficients determined by the canonical angles $\theta_{k}$. Using Eqn. (8) we obtain an alternative expression, viz,

$$
\omega+P=\sum_{k=1}^{m} \frac{1}{\cos \theta_{k}}\left|\eta_{k}\right\rangle\left\langle\xi_{k}\right| .
$$

\section{The canonical effective Hamiltonian}

Hermitian effective Hamiltonians have independently been introduced by various authors since 1929, when Van Vleck [14, 15, 20] introduced a unitary transformation $\tilde{H}=$ $\exp (-S) H \exp (S)$ to decouple the model space to second order in the interaction. In 1963, Primas [21] considered an order by order expansion of this $\tilde{H}$ using the Baker-CampbellHausdorff formula and commutator functions to determine $S$, a technique also used in many other settings in which a transformation is in a Lie group, see, e.g., Ref. [22] and references therein. This approach was elaborated by Shavitt and Redmon [7], who were the first to mathematically connect this Hermitian effective Hamiltonian to $H_{\mathrm{eff}}^{\mathrm{BB}}$, as in Eqn. (27) below. In the nuclear physics community, Suzuki [23] has been a strong advocate of Hermitian effective interactions and the $a$-body sub-cluster approximation to the $A$-body effective interaction [3, 11, 23]. Hermiticity in this case is essential.

Even though a Hermitian effective Hamiltonian is not unique due to the non-uniqueness of $S=-S^{\dagger}$, the various Hermitian effective Hamiltonians put forward in the literature all turn out to be equivalent [6]. In the spirit of Klein and Shavitt [6], 7] we employ the term "canonical effective Hamiltonian" since this emphasizes the "natural" and geometric nature of the Hermitian effective Hamiltonian, which we denote by $H_{\mathrm{eff}}^{\mathrm{c}}$.

Recall the spectral decomposition

$$
H_{\mathrm{eff}}^{\mathrm{c}}=\sum_{k=1}^{m} E_{k}\left|\psi_{k}^{\mathrm{eff}}\right\rangle\left\langle\psi_{k}^{\mathrm{eff}}\right|,
$$


where the (orthonormal) effective eigenvectors are now defined by the following optimization property: The effective eigenvectors $\left|\psi_{k}^{\text {eff }}\right\rangle$ are the closest possible to the exact eigenvectors $\left|\psi_{k}\right\rangle$ while still being orthonormal. Thus, where the Bloch-Brandow approach globally minimizes the distance between the eigenvectors, at the cost of non-orthonormality, the canonical approach has the unitarity constraint on the similarity transformation, rendering $H_{\text {eff }}^{\mathrm{c}}$ Hermitian.

Given a collection $\Phi=\left\{\left|\phi_{1}\right\rangle, \ldots,\left|\phi_{m}\right\rangle\right\} \subset \mathcal{P}$ of $m$ vectors, which are candidates for effective eigenvectors, define the functional $S[\Phi]$ by

$$
\begin{aligned}
S[\Phi] & :=\sum_{k=1}^{m} \|\left|\phi_{k}\right\rangle-\left|\psi_{k}\right\rangle \|^{2} \\
& =K+\sum_{k=1}^{m} \| P\left|\psi_{k}\right\rangle-\left|\phi_{k}\right\rangle \|^{2} \\
& =K+\sum_{k=1}^{m} \|\left|\phi_{k}\right\rangle \|^{2}-2 \operatorname{Re} \sum_{k=1}^{m}\left\langle\psi_{k}|P| \phi_{k}\right\rangle,
\end{aligned}
$$

where $K=\sum_{k=1}^{m} \| Q\left|\psi_{k}\right\rangle \|^{2}$ is a constant in this context. The effective eigenvectors are now minimizers of $S[\Phi]$.

The global minimum, when $\Phi \subset \mathcal{P}$ is allowed to vary freely, is attained for $\left|\phi_{k}\right\rangle=\left|P \psi_{k}\right\rangle$, the Bloch-Brandow effective eigenvectors. However, the canonical effective eigenvectors are determined by minimizing $S[\Phi]$ over all orthonormal sets $\Phi$, which then becomes equivalent to maximizing the last term in Eqn. (19), i.e., the overlaps $\sum_{k} \operatorname{Re}\left\langle\psi_{k}|P| \phi_{k}\right\rangle$ under the orthonormality constraint.

We will now prove the striking fact that the solution is given by

$$
\left|\psi_{k}^{\mathrm{eff}}\right\rangle=\left|\phi_{k}\right\rangle=e^{-G}\left|\psi_{k}\right\rangle
$$

where the unitary operator $Z:=\exp (G) \in S U(n)$ is the rotation (12). Equation (20) should be compared with Eqn. (7). Thus, the exact eigenvectors are simply the direct rotations of the effective eigenvectors from the model space into $\mathcal{E}$.

Let us expand $\left|\psi_{k}\right\rangle \in \mathcal{E}$ and $\left|\phi_{k}\right\rangle \in \mathcal{P}$ in the principal vector bases, viz,

$$
\begin{aligned}
\left|\psi_{k}\right\rangle & =\sum_{j=1}^{m}\left|\eta_{j}\right\rangle\left\langle\eta_{j} \mid \psi_{k}\right\rangle, \\
\left|\phi_{k}\right\rangle & =\sum_{j=1}^{m}\left|\xi_{j}\right\rangle\left\langle\xi_{j} \mid \phi_{k}\right\rangle .
\end{aligned}
$$


Using $\left\langle\eta_{j} \mid \xi_{k}\right\rangle=\delta_{j, k} \cos \theta_{j}$, we compute the sum $A:=\sum_{k=1}^{m}\left\langle\psi_{k}|P| \phi_{k}\right\rangle$ as

$$
\begin{aligned}
A & =\sum_{k, j, \ell=1}^{m}\left\langle\psi_{k} \mid \eta_{j}\right\rangle\left\langle\eta_{j} \mid \xi_{\ell}\right\rangle\left\langle\xi_{\ell} \mid \phi_{k}\right\rangle \\
& =\sum_{j, k=1}^{m} \cos \theta_{j}\left\langle\xi_{j} \mid \phi_{k}\right\rangle\left\langle\psi_{k} \mid \eta_{j}\right\rangle
\end{aligned}
$$

Now,

$$
A=\sum_{j=1}^{m} \cos \theta_{j} u_{j, j},
$$

where $u_{j, k}$ is a unitary matrix, which implies $\left|u_{j, j}\right| \leq 1$. Moreover, $u_{j, j}=1$ for all $j$ if and only if $u_{j, k}=\delta_{j, k}$, which then maximizes $A$, and also $\operatorname{Re} A$. Thus,

$$
\sum_{k=1}^{m}\left\langle\xi_{j} \mid \phi_{k}\right\rangle\left\langle\psi_{k} \mid \eta_{\ell}\right\rangle=\delta_{j, \ell}
$$

i.e.,

$$
\left\langle\xi_{j} \mid \phi_{k}\right\rangle=\left\langle\eta_{j} \mid \psi_{k}\right\rangle=\left\langle\xi_{j}\left|Z^{\dagger}\right| \psi_{k}\right\rangle
$$

from which Eqn. (20) follows since $\left\{\left|\xi_{k}\right\rangle\right\}_{k=1}^{m}$ is a basis for $\mathcal{P}$, and the proof is complete.

The similarity transform in Eqn. (5) is thus manifest, with $S=G$, viz,

$$
H_{\mathrm{eff}}^{\mathrm{c}}=P Z^{\dagger} H Z P=P e^{-G} H e^{G} P .
$$

Moreover, $Q \tilde{H} P=P \tilde{H} Q=0$, verifying that the direct rotation in fact block diagonalizes $H$.

\section{E. Computing $\left|\psi_{k}^{\text {eff }}\right\rangle$}

Assume that $\left|P \psi_{k}\right\rangle:=P\left|\psi_{k}\right\rangle, k=1, \cdots, m$ are available. The effective eigenvectors $\left|\psi_{k}^{\text {eff }}\right\rangle$ are then given by a basis change $F$, i.e., the operator $F: \mathcal{P} \rightarrow \mathcal{P}$ defined by

$$
F\left|P \psi_{k}\right\rangle:=\left|\psi_{k}^{\mathrm{eff}}\right\rangle
$$


Using the principal vector basis we obtain

$$
\begin{aligned}
F\left|P \psi_{k}\right\rangle & =F P \sum_{j=1}^{m}\left|\eta_{j}\right\rangle\left\langle\eta_{j} \mid \psi_{k}\right\rangle \\
& =F \sum_{j=1}^{m} \cos \theta_{j}\left|\xi_{j}\right\rangle\left\langle\eta_{j} \mid \psi_{k}\right\rangle \\
& :=\sum_{j=1}^{m}\left|\xi_{j}\right\rangle\left\langle\xi_{j} \mid \psi_{k}^{\text {eff }}\right\rangle \\
& =\sum_{j=1}^{m}\left|\xi_{j}\right\rangle\left\langle\eta_{j} \mid \psi_{k}\right\rangle
\end{aligned}
$$

from which we get the SVD

$$
\begin{aligned}
F & :=\sum_{k=1}^{m} \frac{1}{\cos \theta_{k}}\left|\xi_{k}\right\rangle\left\langle\xi_{k}\right| \\
& =\left(\omega^{\dagger} \omega+P\right)^{1 / 2}
\end{aligned}
$$

where we have used Eqn. (18). From Eqn. (22) we see that $F$ is symmetric and positive definite. Moreover, smaller angles $\theta_{k}$ means $F$ is closer to the identity, consistent with $\mathcal{E}$ is closer to $\mathcal{P}$.

Let $\left|P \psi_{k}\right\rangle$ now be given in the orthonormal "zero order" basis $\left\{\left|e_{k}\right\rangle\right\}_{k=1}^{m}$ for $\mathcal{P}$, i.e., we have the basis change operator $\tilde{U}$ given by

$$
\tilde{U}:=\sum_{k=1}^{m}\left|P \psi_{k}\right\rangle\left\langle e_{k}\right|,
$$

which transforms from the given basis to the Bloch-Brandow effective eigenvectors. In terms of the principal vector basis,

$$
\begin{aligned}
\tilde{U} & =\sum_{j=1}^{m} \cos \theta_{j}\left|\xi_{j}\right\rangle\left\langle\eta_{j}\left|\sum_{k}\right| \psi_{k}\right\rangle\left\langle e_{k}\right| \\
& =: \sum_{j=1}^{m} \cos \theta_{j}\left|\xi_{j}\right\rangle\left\langle y_{j}\right|,
\end{aligned}
$$

which is, in fact, the SVD since the last sum over $k$ is a unitary map from $\mathcal{P}$ to $\mathcal{E}$. In the operator $\tilde{U} \tilde{U}^{\dagger}$ this basis-dependent factor cancels, viz,

$$
\begin{aligned}
\tilde{U} \tilde{U}^{\dagger} & =\sum_{k=1}^{m}\left|P \psi_{k}\right\rangle\left\langle P \psi_{k}\right| \\
& =\sum_{k=1}^{m} \cos ^{2} \theta_{k}\left|\xi_{k}\right\rangle\left\langle\xi_{k}\right|,
\end{aligned}
$$


that is,

$$
F=\left(\tilde{U} \tilde{U}^{\dagger}\right)^{-1 / 2}
$$

If we seek $\left|\psi_{k}^{\text {eff }}\right\rangle$ in the basis $\left\{\left|e_{k}\right\rangle\right\}_{k=1}^{m}$ as well, we let $\tilde{V}$ be the corresponding basis change operator, i.e.,

$$
\tilde{V}:=F \tilde{U}=\left(\tilde{U} \tilde{U}^{\dagger}\right)^{-1 / 2} \tilde{U}
$$

Equation (25) shows that $\left|\psi_{k}^{\text {eff }}\right\rangle$ is obtained by "straightening out" $\left|P \psi_{k}\right\rangle$, and that this depends only on the latter vectors. This is, in fact, an alternative to the common GramSchmidt orthogonalization used in mathematical constructions and proofs. It was first introduced by Löwdin [24] under the name "symmetric orthogonalization", and so-called "Löwdin bases" are widely-used in quantum chemistry, where non-orthogonal basis functions are orthogonalized according to Eqn. (25). It seemingly requires both inversion and matrix square root, but is easily computed using the SVD. Combining Eqns. (22) and (24) gives

$$
\tilde{V}=\sum_{k=1}^{m}\left|\xi_{k}\right\rangle\left\langle y_{k}\right|
$$

so that if the SVD (24) is available, $\tilde{V}$ is readily computed. Eqn. (26) is easily expressed in terms of matrices, but we defer the discussion to Sec. IV.

\section{F. Shavitt's expression for $\exp (G)$}

Shavitt and Redmon [7] proved that

$$
G=\tanh ^{-1}\left(\omega-\omega^{\dagger}\right)
$$

gives the Lie algebra element for the unitary operator $Z=\exp (G)$. The quite complicated proof was done using an expansion of the similarity transform using the Baker-CampbellHausdorff formula.

It may be clear now, that in the present context we obtain the result simply as a byproduct of the treatment in Section $11 \mathrm{~B}$ and the SVD (17) of $\omega$, given in terms of the principal vectors and angles. We prove this here.

The function $\tanh ^{-1}(z)$ is defined by its (complex) Taylor expansion about the origin, i.e.,

$$
\tanh ^{-1}(z)=\sum_{n=0}^{\infty} \frac{z^{2 n+1}}{2 n+1}
$$


The series converges for $|z|<1$. Moreover,

$$
\tanh ^{-1}(z)=\frac{1}{2} \ln \left(\frac{1+z}{1-z}\right)
$$

also valid for $|z|<1$. For $z:=\omega-\omega^{\dagger}$ we compute

$$
z=\sum_{k=1}^{m} \mu_{k}\left(\left|\chi_{k}\right\rangle\left\langle\xi_{k}|-| \xi_{k}\right\rangle\left\langle\chi_{k}\right|\right), \quad \mu_{k}:=\tan \left(\theta_{k}\right)
$$

Using orthogonality relations between $\left|\xi_{k}\right\rangle$ and $\left|\chi_{k}\right\rangle$ we obtain

$$
\begin{aligned}
z^{2 n+1} & =(-1)^{n} \sum_{k=1}^{m} \mu_{k}^{2 n+1}\left(\left|\chi_{k}\right\rangle\left\langle\xi_{k}|-| \xi_{k}\right\rangle\left\langle\chi_{k}\right|\right) \\
& =i \sum_{k=1}^{m}\left(-i \mu_{k}\right)^{2 n+1}\left(\left|\chi_{k}\right\rangle\left\langle\xi_{k}|-| \xi_{k}\right\rangle\left\langle\chi_{k}\right|\right) .
\end{aligned}
$$

Using $i \tanh ^{-1}(-i z)=\tan ^{-1}(z)$, we sum the series (28) to

$$
G=\tanh ^{-1}(z)=\sum_{k=1}^{m} \theta_{k}\left(\left|\chi_{k}\right\rangle\left\langle\xi_{k}|-| \xi_{k}\right\rangle\left\langle\chi_{k}\right|\right),
$$

which is identical to Eqn. (13). The series does not converge for $\theta_{k} \geq \pi / 4$, but the result is trivially analytically continued to arbitrary $0 \leq \theta_{k} \leq \pi / 2$.

We now turn to the effective Hamiltonian. It is common [2, 3, 11] to compute $H_{\mathrm{eff}}^{\mathrm{c}}$ in terms of $\omega$ directly, using the definition (29) of $\tanh ^{-1}(z)$, which implies

$$
e^{ \pm \tanh ^{-1}(z)}=\sqrt{\frac{1 \pm z}{1 \mp z}}=\frac{1 \pm z}{\sqrt{1-z^{2}}},
$$

Upon insertion into $\tilde{H}=\exp (-G) H \exp (G)$, we obtain

$$
\tilde{H}=\frac{1-\omega+\omega^{\dagger}}{\sqrt{1+\omega^{\dagger} \omega+\omega \omega^{\dagger}}} H \frac{1+\omega-\omega^{\dagger}}{\sqrt{1+\omega^{\dagger} \omega+\omega \omega^{\dagger}}} .
$$

Projecting onto $\mathcal{P}$, the effective Hamiltonian becomes

$$
H_{\mathrm{eff}}=\left(P+\omega^{\dagger} \omega\right)^{-1 / 2}\left(P+\omega^{\dagger}\right) H(P+\omega)\left(P+\omega^{\dagger} \omega\right)^{-1 / 2}
$$

By using the Bloch-equation ([6) for the Bloch-Brandow effective Hamiltonian, we may eliminate $Q H Q$ from the above expression for $H_{\text {eff }}$, yielding

$$
H_{\mathrm{eff}}^{\mathrm{c}}=\left(P+\omega^{\dagger} \omega\right)^{1 / 2} H(P+\omega)\left(P+\omega^{\dagger} \omega\right)^{-1 / 2} .
$$


This expression is commonly implemented in numerical applications [3, 25]. By comparing with Eqns. (22) and (16) we immediately see that

$$
H_{\mathrm{eff}}^{\mathrm{c}}=F H_{\mathrm{eff}}^{\mathrm{BB}} F^{-1}
$$

which gives $H_{\mathrm{eff}}^{\mathrm{c}}$ as a similarity transform of $H_{\mathrm{eff}}^{\mathrm{BB}}$. In themselves, Eqns. (31) and (32) are not manifestly Hermitian, stemming from the elimination of $Q H Q$. An implementation would require complicated matrix manipulations, including a matrix square root. It is therefore better to compute $H_{\text {eff }}^{\mathrm{c}}$ using

$$
H_{\mathrm{eff}}^{\mathrm{c}}=\sum_{k} E_{k}\left|\psi_{k}^{\mathrm{eff}}\right\rangle\left\langle\psi_{k}^{\mathrm{eff}}\right|
$$

together with Eqn. (26), where the most complicated operation is the SVD of the operator $\tilde{U}$ given by Eqn. (23). In Sec. [V] we give a concrete matrix expression for $H_{\text {eff }}^{\text {c }}$.

\section{G. Commuting observables}

Great simplifications arise in the general quantum problem if continuous symmetries of the Hamiltonian can be identified, i.e., if one can find one or more observables $S$ such that $[H, S]=0$. Here, we discuss the impact of such symmetries of $H$ on the effective Hamiltonian $H_{\text {eff }}$; both in the Bloch-Brandow and the canonical case. We point out the importance of choosing a model space that is an invariant of $S$ as well, i.e., $[S, P]=0$. In fact, we prove that this is the case if and only if $\left[H_{\text {eff }}, S\right]=0$, i.e., $H_{\text {eff }}$ has the same continuous symmetry.

Let $S=S^{\dagger}$ be an observable such that $[H, S]=0$, i.e., $H$ and $S$ have a common basis of eigenvectors. We shall assume that $\left\{\left|\psi_{k}\right\rangle\right\}_{k=1}^{n}$ is such a basis, viz,

$$
\begin{aligned}
H\left|\psi_{k}\right\rangle & =E_{k}\left|\psi_{k}\right\rangle \\
S\left|\psi_{k}\right\rangle & =s_{k}\left|\psi_{k}\right\rangle .
\end{aligned}
$$

In general, there will be degeneracies in both $E_{k}$ and $s_{k}$.

We now make the important assumption that

$$
[S, P]=0
$$

which is equivalent to

$$
S=P S P+Q S Q .
$$


Under the assumption (34), we have

$$
S\left|P \psi_{k}\right\rangle=P S\left|\psi_{k}\right\rangle=s_{k}\left|P \psi_{k}\right\rangle,
$$

so that the Bloch-Brandow effective eigenvectors are still eigenvectors of $S$ with the same eigenvalue $s_{k}$. Moreover, as we assume that $\left\{\left|P \psi_{k}\right\rangle\right\}_{k=1}^{m}$ is a (non-orthonormal) basis for $\mathcal{P}$, this not possible if $[S, P] \neq 0$. Thus, $\left[H_{\mathrm{eff}}^{\mathrm{BB}}, S\right]=0$ if and only if $[S, P]=0$ (in addition to the assumption (33).)

The assumption (34) also implies that $[S, \omega]=0$, where $\omega=Q \omega P$ is the de-coupling operator. We prove this by checking that it holds for all $\left|\psi_{k}\right\rangle$. For $k \leq m$,

$$
\omega S\left|\psi_{k}\right\rangle=s_{k} Q\left|\psi_{k}\right\rangle=S Q\left|\psi_{k}\right\rangle=S \omega\left|\psi_{k}\right\rangle,
$$

while for $k>m$ we need to expand $P\left|\psi_{k}\right\rangle$ in $\left|P \psi_{j}\right\rangle, j \leq m$, viz,

$$
P\left|\psi_{k}\right\rangle=\sum_{j=1}^{m}\left|P \psi_{j}\right\rangle\left\langle\widetilde{P \psi_{j}}|P| \psi_{k}\right\rangle, \quad k>m
$$

and use Eqn. (35). Furthermore, $\left[S, \omega^{\dagger}\right]^{\dagger}=\left[\omega^{\dagger}, S\right]=0$. It follows that

$$
\left[S,\left(\omega-\omega^{\dagger}\right)^{n}\right]=0, \quad n=0,1, \ldots,
$$

and, by Eqn. (28), that

$$
\left[S, e^{G}\right]=\left[S, e^{-G}\right]=0 .
$$

This gives

$$
S\left|\psi_{k}^{\mathrm{eff}}\right\rangle=S e^{-G}\left|\psi_{k}\right\rangle=s_{k}\left|\psi_{k}^{\mathrm{eff}}\right\rangle .
$$

Again, since $\left\{\left|\psi_{k}^{\mathrm{e}}\right\rangle\right\}_{k=1}^{m}$ is a basis for $\mathcal{P}$, this holds if and only if $[S, P]=0$. Accordingly, $\left[H_{\mathrm{eff}}^{\mathrm{c}}, S\right]=0$ if and only if $[S, P]=0$ (and the assumption (33).)

The importance of this fact is obvious. If one starts with a Hamiltonian that conserves, say, angular momentum, and computes the effective interaction using a model space that is not an invariant for the angular momentum operator, i.e., not rotationally symmetric, then the final Hamiltonian will not have angular momentum as a good quantum number.

One possible remedy if $[P, S] \neq 0$ is to define the effective observable $S_{\text {eff }}:=$ $P \exp (-G) S \exp (G) P$ (which in the commuting case is equal to $P S P$ ) which obviously commutes with $H_{\text {eff }}$ and satisfies

$$
S_{\text {eff }}\left|\psi_{k}^{\mathrm{eff}}\right\rangle=s_{k}\left|\psi_{k}^{\mathrm{eff}}\right\rangle .
$$


This amounts to modifying the concept of rotational symmetry in the above example.

The assumptions (33) and (34) have consequences also for the structure of the principal vectors $\left|\xi_{k}\right\rangle \in \mathcal{P}$ and $\left|\eta_{k}\right\rangle \in \mathcal{E}$. Indeed, write

$$
\begin{aligned}
\mathcal{E} & =\bigoplus_{s} \mathcal{E}_{s} \\
\mathcal{P} & =\bigoplus_{s} \mathcal{P}_{s}
\end{aligned}
$$

where the sum runs over all distinct eigenvalues $s_{k}, k=1, \cdots, m$ of $S$, and where $\mathcal{E}_{s}\left(\mathcal{P}_{s}\right)$ is the corresponding eigenspace, i.e.,

$$
\begin{aligned}
& \mathcal{E}_{s}:=\operatorname{span}\left\{\left|\psi_{k}\right\rangle: S\left|\psi_{k}\right\rangle=s\left|\psi_{k}\right\rangle\right\} \\
& \mathcal{P}_{s}:=\operatorname{span}\left\{\left|\psi_{k}^{\text {eff }}\right\rangle: S\left|\psi_{k}^{\text {eff }}\right\rangle=s\left|\psi_{k}^{\text {eff }}\right\rangle\right\}
\end{aligned}
$$

The eigenspaces are all mutually orthogonal, viz, $\mathcal{E}_{s} \perp \mathcal{E}_{s^{\prime}}, \mathcal{P}_{s} \perp \mathcal{P}_{s^{\prime}}$, and $\mathcal{E}_{s} \perp \mathcal{P}_{s^{\prime}}$, for $s \neq s^{\prime}$. The definition (3) of the principal vectors and angles can then we written

$$
\begin{aligned}
\cos \left(\theta_{k}\right) & =\max _{s} \max _{\substack{|\xi\rangle \in \mathcal{P}_{s},\langle\xi \mid \xi\rangle=1 \\
\left\langle\xi_{j} \mid \xi\right\rangle=0, j<k}} \max _{\substack{|\eta\rangle \in \mathcal{E}_{s},\langle\eta \eta \eta\rangle=1 \\
\left\langle\eta_{j} \mid \eta\right\rangle=0, j<k}} \operatorname{Re}\langle\xi \mid \eta\rangle \\
& =:\left\langle\xi_{k} \mid \eta_{k}\right\rangle,
\end{aligned}
$$

Thus, for each $k$, there is an eigenvalue $s$ of $S$ such that

$$
\begin{aligned}
& S\left|\xi_{k}\right\rangle=s\left|\xi_{k}\right\rangle \\
& S\left|\eta_{k}\right\rangle=s\left|\eta_{k}\right\rangle,
\end{aligned}
$$

showing that the principal vectors are eigenvectors of $S$ if and only if $[S, P]=0,[S, H]=0$, and the assumption (33).

The present symmetry considerations imply that model spaces obeying as many symmetries as possible should be favored over less symmetric model spaces, since these other model spaces become less "natural" or "less effective" in the sense that their geometry is less similar to the original Hilbert space. This is most easily seen from the fact that principal vectors are eigenvectors for the conserved observable $S$. This may well have great consequences for the widely-used sub-cluster approximation to the effective Hamiltonian in no-core shell model calculations [3, 6, 26], where one constructs the effective Hamiltonian for a system of $a$ particles in order to obtain an approximation to the $A>a$-body effective Hamiltonian. 
The model space in this case is constructed in different ways in different implementations. Some of these model spaces may therefore be better than others due to different symmetry properties.

\section{MATRIX FORMULATIONS}

\section{A. Preliminaries}

Since computer calculations are invariably done using matrices for operators, we here present matrix expressions for $H_{\mathrm{eff}}^{\mathrm{c}}$ and compare them to those usually programmed in the literature, as well as expressions for $H_{\mathrm{eff}}^{\mathrm{BB}}$ and $\omega$.

Recall the standard basis $\left\{\left|e_{k}\right\rangle\right\}_{k=1}^{n}$ of $\mathcal{H}$, where the $\left\{\left|e_{k}\right\rangle\right\}_{k=1}^{m}$ constitute a basis for $\mathcal{P}$. These are usually eigenvectors of the unperturbed "zero order" Hamiltonian $H_{0}$, but we will not use this assumption. As previously we also assume without loss that the eigenpairs we wish to approximate in $H_{\text {eff }}$ are $\left\{\left|\psi_{k}\right\rangle\right\}_{k=1}^{m}$.

An operator $A: \mathcal{H} \rightarrow \mathcal{H}$ has a matrix $\mathrm{A} \in \mathbb{C}^{n \times n}$ associated with it. The matrix elements are given by $\mathrm{A}_{j k}=\left\langle e_{j}|A| e_{k}\right\rangle$ such that

$$
A=\sum_{j, k=1}^{n}\left|e_{j}\right\rangle\left\langle e_{j}|A| e_{k}\right\rangle\left\langle e_{k}\left|=\sum_{j, k=1}^{n}\right| e_{j}\right\rangle \mathrm{A}_{j k}\left\langle e_{k}\right| .
$$

Similarly, any vector $|\phi\rangle \in \mathcal{H}$ has a column vector $\vec{\phi} \in \mathbb{C}^{n}$ associated with it, with $\vec{\phi}_{j}=$ $\left\langle e_{j} \mid \phi\right\rangle$. We will also view dual vectors, e.g., $\langle\psi|$, as row vectors.

The model space $\mathcal{P}$ and the excluded space $\mathcal{Q}$ are conveniently identified with $\mathbb{C}^{m}$ and $\mathbb{C}^{n-m}$, respectively. Also note that $P A P, P A Q$, etc., are identified with the upper left $m \times m$, upper right $m \times(n-m)$, etc., blocks of A as in Eqn. (1). We use a notation inspired by FORTRAN and MATLAB and write

$$
\mathrm{PAP}=\mathrm{A}(1: m, 1: m), \quad \mathrm{PAQ}=\mathrm{A}(1: m, m+1: n)
$$

and so forth.

We introduce the unitary operator $U$ as

$$
U=\sum_{k=1}^{n}\left|\psi_{k}\right\rangle\left\langle e_{k}\right|
$$


i.e., a basis change from the chosen standard basis to the eigenvector basis. The columns of $\mathrm{U}$ are the eigenvectors' components in the standard basis, i.e.,

$$
\mathrm{U}_{j k}=\vec{\psi}_{k, j}=\left\langle e_{j} \mid \psi_{k}\right\rangle
$$

and are typically the eigenvectors returned from a computer implementation of the spectral decomposition, viz,

$$
\mathrm{H}=\mathrm{UEU}^{\dagger}, \quad \mathrm{E}=\operatorname{diag}\left(E_{1}, \ldots, E_{n}\right) .
$$

The SVD is similarly transformed to matrix form. The SVD defined in Sec. 【IB is then formulated as follows: For any matrix $\mathrm{A} \in \mathbb{C}^{q \times r}$ there exist matrices $\mathbf{X} \in \mathbb{C}^{q \times p}(p=\min (q, r))$ and $\mathrm{Y} \in \mathbb{C}^{r \times p}$, such that $\mathrm{X}^{\dagger} \mathrm{X}=\mathrm{Y}^{\dagger} \mathrm{Y}=I_{p}$ (the identity matrix $\mathbb{C}^{p \times p}$ ), and a non-negative diagonal matrix $\Sigma \in \mathbb{R}^{p \times p}$ such that

$$
A=X \Sigma Y^{\dagger}
$$

Here, $\Sigma=\operatorname{diag}\left(\sigma_{1}, \cdots, \sigma_{p}\right), \sigma_{k}$ being the singular values.

The columns of $\mathbf{X}$ are the left singular vectors' components, i.e., $\mathbf{X}_{j, k}=\left\langle e_{j} \mid x_{k}\right\rangle$, and similarly for $\mathrm{Y}$ and the right singular vectors. The difference between the two SVD formulations is then purely geometric, as the matrix formulation favorizes the standard bases in $\mathcal{X}$ and $\mathcal{Y}$.

The present version of the matrix SVD is often referred to as the "economic" SVD, since the matrices $X$ and $Y$ may be extended to unitary matrices over $\mathbb{C}^{q}$ and $\mathbb{C}^{r}$, respectively, by adding singular values $\sigma_{k}=0, k>m$. The matrix $\Sigma$ is then a $q \times r$ matrix with "diagonal" given by $\sigma_{k}$. This is the "full" SVD, equivalent to our basis-free definition.

\section{B. Algorithms}

Let the $m$ eigenvectors $\left|\psi_{k}\right\rangle$ be calculated and arranged in a matrix $\mathrm{U}$, i.e., $\psi_{k}=\mathrm{U}(1: n, k)$ (where the subscript does not pick a single component). Consider the operator $\tilde{U}$ defined in Eqn. (23), whose matrix' columns are the Bloch-Brandow effective eigenvectors $\left|P \psi_{k}\right\rangle$ in the standard basis, viz,

$$
\tilde{\mathrm{U}}=\mathrm{U}(1: m, 1: m)
$$

The columns of the matrix of $\tilde{V}=\left(\tilde{U} \tilde{U}^{\dagger}\right)^{-1 / 2} \tilde{U}$ are the canonical effective eigenvectors $\vec{\psi}_{k}^{\text {eff }}$. The SVD (24) can be written

$$
\tilde{U}=X \Sigma Y^{\dagger}
$$


which gives

$$
\tilde{U} \tilde{U}^{\dagger}=X \Sigma^{2} X^{\dagger}
$$

Since $\Sigma_{k k}=\cos \theta_{k}>0$, we obtain

$$
\left(\tilde{U} \tilde{U}^{\dagger}\right)^{-1 / 2}=X \Sigma^{-1} X^{\dagger}
$$

which gives, when applied to U

$$
\tilde{V}=\left(\tilde{U} \tilde{U}^{\dagger}\right)^{-1 / 2} \tilde{U}=X Y^{\dagger}
$$

Thus, we obtain the canonical effective eigenvectors by taking the matrix SVD of $\tilde{U}=\mathrm{U}(1$ : $m, 1: m)$ and multiplying together the matrices of singular vectors. As efficient and robust SVD implementations are almost universally available, e.g., in the LAPACK library, this makes the canonical effective interaction much easier to compute compared to Eqn. (31), viz,

$$
\mathrm{H}_{\mathrm{eff}}^{\mathrm{c}}=\tilde{\mathrm{V}} \mathrm{E}(1: m, 1: m) \tilde{\mathrm{V}}^{\dagger}
$$

This version requires one SVD computation and three matrix multiplications, all with $m \times m$ matrices, one of which is diagonal. Equation (31) requires, on the other hand, several more matrix multiplications, inversions and the square root computation. The Bloch-Brandow effective Hamiltonian is simply calculated by

$$
\mathrm{H}_{\mathrm{eff}}^{\mathrm{BB}}=\tilde{\mathrm{U}} \mathrm{E}(1: m, 1: m) \tilde{\mathrm{U}}^{-1}
$$

For the record, the matrix of $\omega$ is given by

$$
\boldsymbol{\omega}=\mathrm{U}(m+1: n, 1: m) \tilde{\mathrm{U}}^{-1}
$$

although we have no use for it when using the SVD based algorithm. It may be useful, though, to be able to compute the principal vectors for $\mathcal{P}$ and $\mathcal{E}$. For this, one may compute the SVD of $\omega$ or of $\mathrm{PP}^{\prime}=\tilde{U} U(1: n, 1: m)^{\dagger}$, the latter which gives $\cos \theta_{k},\left|\xi_{k}\right\rangle$ and $\left|\eta_{k}\right\rangle$ directly in the standard basis as singular values and vectors, respectively.

\section{DISCUSSION AND AND OUTLOOK}

We have characterized the effective Hamiltonians commonly used in nuclear shell-model calculations in terms of geometric properties of the spaces $\mathcal{P}$ and $\mathcal{E}$. The SVD and the 
principal angles and vectors were central in the investigation. While the Bloch-Brandow effective Hamiltonian is obtained by orthogonally projecting $\mathcal{E}$ onto $\mathcal{P}$, thereby globally minimizing the norm-error of the effective eigenvectors, the canonical effective Hamiltonian is obtained by rotating $\mathcal{E}$ into $\mathcal{P}$ using $\exp (-G)$, which minimizes the norm-error while retaining orthonormality of the effective eigenvectors. Moreover, we obtained a complete description of the de-coupling operator $\omega$ in terms of the principal angles and vectors defining $\exp (G)$.

An important question is whether the present treatment generalizes to infinite dimensional Hilbert spaces. Our analysis fits into the general assumptions in the literature, being that $n=\operatorname{dim}(\mathcal{H})$ is large but finite, or at least that the spectrum of $H$ purely discrete. A minimal requirement is that $H$ has $m$ eigenvalues, so that $\mathcal{E}$ can be constructed. In particular, the SVD generalizes to finite rank operators in the infinite dimensional case, and are thus valid for all the operators considered here even when $n=\infty$.

Unfortunately, $H$ has almost never a purely discrete spectrum. It is well-known that the spectrum in general has continuous parts and resonances embedded in these, and a proper theory should treat these cases as well as the discrete part. In fact, the treatments of $H_{\text {eff }}$ in the literature invariably glosses over this. It is an interesting future project to develop a geometric theory for the effective Hamiltonians which incorporates resonances and continuous spectra.

The geometrical view simplified and unified the available treatments in the literature somewhat, and offered further insights into the effective Hamiltonians. Moreover, the the symmetry considerations in Sec. IIIG may have significant bearing on the analysis of perturbation expansions and the properties of sub-cluster approximations to $H_{\mathrm{eff}}^{\mathrm{c}}$.

Indeed, it is easy to see, that if we have a complete set of commuting observables (CSCO) [27] for $H_{0}$, and the same set of observables form a CSCO for $H_{1}$, all eigenvalues and eigenfunctions of $H(z)=H_{0}+z H_{1}$ are analytic in $z \in \mathbb{C}$, implying that the RayleighSchroedinger perturbation series for $H=H_{0}+H_{1}$ converges (i.e., at $z=1$ ) [28]. Intuitively, the fewer commuting observables we are able to identify, the more likely it is that there are singularities in $H_{\text {eff }}(z)$, so called intruder states. The Rayleigh-Schroedinger series diverges outside the singularity closest to $z=0$ [28], and in nuclear systems this singularity is indeed likely to be close to $z=0$. On the other hand, resummation of the series can be convergent and yield an analytic continuation of $H_{\text {eff }}$ outside the region of convergence [29]. To the 
author's knowledge, there is no systematic treatment of this phenomenon in the literature. On the contrary, to be able to do such a resummation is sort of a "holy grail" of many-body perturbation theory. A geometric study of the present kind to many-body perturbation theory and diagram expansions may yield a step closer to this goal, as we have clearly identified the impact of commuting observables on the principal vectors of $\mathcal{E}$ and $\mathcal{P}$.

We have also discussed a compact algorithm in terms of matrices to compute $H_{\text {eff }}^{\mathrm{c}}$, relying on the SVD. To the author's knowledge, this algorithm is previously unpublished. Since robust and fast SVD implementations are readily available, e.g., in the LAPACK library, and since few other matrix manipulations are needed, it should be preferred in computer implementations.

As stressed in the Introduction, the algorithms presented are really only useful if we compute the exact effective Hamiltonian, as opposed to a many-body perturbation theoretical calculation, and if we know what exact eigenpairs to use, such as in a sub-cluster approximation. In this case, one should analyze the error in the approximation, i.e., the error in neglecting the many-body correlations in $H_{\mathrm{eff}}^{\mathrm{c}}$. In the perturbative regime, some results exist [6]. The author believes, that the geometric description may facilitate a deeper analysis, and this is an interesting idea for future work.

\section{Acknowledgments}

The author wishes to thank Prof. M. Hjorth-Jensen, CMA, for helpful discussions. This work was funded by CMA through the Norwegian Research Council.

[1] D. Dean, T. Engeland, M. Hjorth-Jensen, M. Kartamyshev, and E. Osnes, Prog. Part. Nucl. Phys. 53, 419 (2004).

[2] E. Caurier, G. Martinez-Pinedo, F. Nowacki, A. Poves, and A. P. Zuker, Rev. Mod. Phys. 77, 427 (pages 62) (2005).

[3] P. Navrátil, J. Vary, and B. Barrett, Phys. Rev. C 62, 054311 (2000).

[4] C. Bloch, Nucl. Phys. 6, 329 (1958).

[5] B. Brandow, Rev. Mod. Phys 39, 771 (1967).

[6] D. Klein, J. Chem. Phys. 61, 786 (1974). 
[7] I. Shavitt and L. T. Redmon, The Journal of Chemical Physics 73, 5711 (1980).

[8] I. Lindgren, J. Phys. B: At. Molec. Phys. 7, 2441 (1974).

[9] P. Ellis and E. Osnes, Rev. Mod. Phys. 49, 777 (1977).

[10] I. Lindgren and J. Morrison, Atomic Many-Body Theory (Springer, 1982).

[11] K. Suzuki, Prog. Theor. Phys. 68, 246 (1982).

[12] T. Kuo, Springer Lecture Notes In Physics 144, 248 (1981).

[13] F. Andreozzi, Phys. Rev. C 54, 684 (1996).

[14] J. Van Vleck, Phys. Rev. 33, 467 (1929).

[15] Kemble, The Fundamental Principles of Quantum Mechanics with Elementary Applications (McGraw Hill, 1937).

[16] A. Björck and G. Golub, Math. Comp. 27, 579 (1973).

[17] A. Knyazev and M. Argentati, SIAM J. Sci. Comput. 23, 2008 (2002).

[18] G. Golub and C. van Loan, Matrix computations (Johns Hopkins, 1989).

[19] C. Davis and W. M. Kahan, SIAM Journal on Numerical Analysis 7, 1 (1970).

[20] O. Jordahl, Phys. Rev. 45, 87 (1934).

[21] H. Primas, Rev. Mod. Phys. 35, 710 (1963).

[22] S. Blanes, F. Casas, J. Oteo, and J. Ros, J. Phys. A: Math. Gen. 31, 259 (1998).

[23] K. Suzuki, Prog. Theor. Phys. 68, 1627 (1982).

[24] P.-O. Löwdin, The Journal of Chemical Physics 18, 365 (1950).

[25] G. Hagen, M. Hjorth-Jensen, and N. Michel, Physical Review C (Nuclear Physics) 73, 064307 (pages 13) (2006).

[26] J. Da Providencia and C. Shakin, Ann. Phys. (N.Y.) 30, 95 (1964).

[27] A. Messiah, Quantum Mechanics (Dover, 1999).

[28] T. Schucan and H. Weidenmüller, Ann. Phys. 76, 483 (1973).

[29] P. Schaefer, Ann. Phys. 87, 375 (1974). 